\title{
Kategorisierung, Kontrolle, Vertrauen? Arbeits- und Identitätsdokumente im 19. und frühen 20. Jahrhundert ${ }^{1}$
}

\author{
Sigrid Wadauer
}

\section{Abstract}

From the mid-19th century until the 1920s, legal employment in many areas of wage labour or domestic services within the realm of the Habsburg monarchy or in Austria required the worker or servant to present an employment record book (either an Arbeits- or a Dienstbotenbuch). Unlike in Germany or France, the requirement to possess such work, identity and travel documents was extended to more and more categories of gainful employment. Public authorities and employers perceived these as an indispensable prerequisite for maintaining security and order. In the publications of labour and domestic servant organisations, by contrast, these documents were characterized primarily as instruments of control, compulsion and as symbols of humiliation. However, these employment record books can also be viewed as attesting to an increasing, albeit unequal, formalisation and regulation of labour and employment relationships. They documented occupations and employment relationships of workers and domestic servants that involved a widely disparate range of rights and obligations. This contribution outlines how the various participants discussed, used, misused or avoided these documents and, depending on their position and perspective, the various kinds of protections and risks connected with them.

\section{Einleitung}

Bei der „Ausübung des Polizei- und Gendarmeriedienstes zur Aufrechterhaltung der öffentlichen Ordnung, Ruhe und Sicherheit“, so ein burgenländischer ${ }^{2}$ Gendarmerierevierinspektor 1926, wurden täglich hunderte „bedenkliche Personen“ angehalten und Unbekannte zur Legitimierung aufgefordert (Krautsieder 1926, S. 4). Die in diesem Zusammenhang von

1 Der Text basiert auf Forschungen, die von der Gerda-Henkel-Stiftung (AZ 52/V/18) und im Anschluss daran vom Austrian Science Fund (FWF, Projektnr. P32226G29) gefördert wurden bzw. werden. Ich danke Jessica Richter und Peter Haslinger für Diskussion, Kritik und Hinweise.

2 Das Gebiet des heutigen Burgenlandes gehörte bis zum Ende des Ersten Weltkrieges zur ungarischen Reichshälfte der Habsburgermonarchie. 
verschiedenen Personen vorgewiesenen Ausweisdokumente waren von bemerkenswerter Vielfalt. Der Autor nennt Reisepässe, Grenzverkehrsscheine und Identitätsbescheinigungen im Kontext von Grenzübertritten. In Gebrauch waren weiters Hausierbücher, Dienstkarten für Hausangestellte, Dienstbotenbücher, Ausweiskarten für gewerbliche Hilfsarbeiter, Arbeitsbücher, Arbeitskarten für Kinder unter 14 Jahren, Legitimationskarten für Handlungsreisende, Lizenzen für Wandergewerbe, Gewerbescheine, Konzessionsurkunden, Waffenpässe, Heimatscheine, Geburts- und Taufscheine, Trauungsscheine, Führerscheine, Besitzbögen, Steuerdekrete, Legitimationen und Bestätigungen der Militärbehörden, Eisenbahnlegitimationen und anderes mehr. „Die den Sicherheitsorganen vorgewiesenen Ausweisdokumente" variierten nach (oft geschlechtsspezifischem) Erwerb oder Beruf, nach Alter, staatlicher Zugehörigkeit und anderem mehr, sie konnten „richtig oder auch falsch sein.“ Richtig, so Revierinspektor Krautsieder, wäre ein Ausweisdokument dann, wenn es von einer Behörde für den oder die Vorzeigende* $n$ ausgestellt worden war und korrekte Personaldaten enthielt. Allerdings waren nur wenige der genannten Dokumente mit einem Lichtbild ausgestattet. Häufig waren die vorgewiesenen Papiere in schlechtem Zustand, abgenützt und „verstümmelt“, also zur „Sicherstellung der Identität" nur bedingt geeignet. Manche reisten unter falschem Namen, mit fremden Dokumenten. Zudem würden sich seit Entstehen der Nachfolgestaaten der Monarchie auch viele Personen (nun aus dem Ausland) mit fragwürdigen Papieren herumtreiben, die grenzüberscheitende Überprüfung solcher Dokumente wäre besonders schwierig; die „Ausweisunsicherheit" hätte sich also noch verschlechtert. Gerade auf dem Land wären Bevölkerung und Gemeindebehörden allzu leichtgläubig.

Die Möglichkeiten (und auch die Zwänge) sich auszuweisen variierten also in verschiedener Hinsicht, und sie konnten sich verändern. Wir haben es nicht bloß mit einer Differenz - bedenklich/unbedenklich -, sondern mit einer Reihe von Eigenschaften von unterschiedlichem Gewicht in einer Bandbreite an Konstellationen zu tun. Staatsangehörigkeit war dabei ein Aspekt unter vielen (und nicht immer per se der wichtigste). Ausweise dokumentierten mit mehr oder minder großer Zuverlässigkeit die je unterschiedlichen Personalien, das Aussehen und die staatliche bzw. heimatrechtliche Zugehörigkeit ${ }^{3}$ einer Person. Aber auch die Art der Dokumente selbst, ihr Zustand, beschrieb und kategorisierte ihre Besitzer*innen und ließ sie mehr oder minder vertrauenswürdig oder bedenklich erscheinen.

3 Das Heimatrechtsprinzip blieb in Österreich bis 1939 bestehen. Es begründete das Recht auf ungestörten Aufenthalt und Armenhilfe. RGBl. 1863/105. 
Ausweise stellten Eigenschaften und Unterschiede fest und konnten zu deren Herstellung beitragen; sie dienten einem „social sorting“ (Lyon 2009). Dies war allerdings nicht nur eine Sache der Polizei. In die Produktion, Kontrolle und Benutzung von Dokumenten war eine Reihe von Behörden, Körperschaften und andere Parteien involviert. Ausweisdokumente kamen in amtlichen wie nicht-amtlichen Kontexten zum Einsatz und dienten verschiedenen - mehr oder minder legitimen - Zwecken (vgl. Greefs/Winter 2019; About et al. 2013; Breckenridge/Szreter 2012; Higgs 2011; Groebner 2004; Caplan/Torpey 2001). Über Sicherheit(en) und Gefahren herrschte also kein Konsens, das ist an Arbeits- und Dienstbotenbüchern, die im Folgenden in den Mittelpunkt gestellt werden, gut zu verdeutlichen. ${ }^{4}$

Arbeits- und Dienstbotenbücher waren in der Habsburgermonarchie bzw. in Österreich von der Mitte des 19. Jahrhunderts bis in die 1920er Jahren weit verbreitet. Sie waren eine Voraussetzung für eine legale Beschäftigung in vielen Arten des Erwerbs, sie konnten als Arbeits-, Identitäts- und Reisedokumente im In- und Ausland benutzt werden (vgl. Morgenstern 1912, S. 120-128; Heller 1908, S. 604-627; Rosenbaum 1908; Mayerhofer's Handbuch, 1897). Die Vorschrift, solche Dokumente zu besitzen, wurde - anders als etwa in den deutschen Ländern oder Frankreich (vgl. dazu etwa Landriani 2019; Lehnert 2017; Keiser 2013; Reineke 2010; Stieda 1890) - im Laufe des 19. Jahrhunderts auf immer mehr Kategorien der Erwerbstätigkeit ausgedehnt. Die Dokumente waren in beiden Reichshälften der Habsburgermonarchie in Gebrauch, ich beziehe mich im Folgenden aber ausschließlich auf die Politik und Gesetzgebung der im Reichsrat vertretenen Königreiche und Länder, also auf die österreichische Reichshälfte. Mussten zunächst, nach der Gewerbeordnung von 1859, nur Gesellen und Fabrikarbeiter (Professionisten) und „weibliche Hilfsarbeiter" in gleichen Dienstverhältnissen solche Arbeitsbücher besitzen, gleich ob sie wanderten oder nicht ${ }^{5}$, so waren sie seit 1866 auch für Bergarbeiter vorgeschrieben, seit 1885 für alle „Arbeitspersonen, welche bei Gewerbsunternehmungen in regelmäßiger Beschäftigung"standen - also auch für

4 Zu Aspekten von Sicherheit vgl. Conze 2018; Daase 2012; Conze 1984.

5 RGBl. 1859/227, $\$ 74$ und Anhang. Diese Arbeitsbücher ersetzten die Wanderbücher, welche die Kundschaften ersetzten. Vgl. Patent vom 24. Februar 1827. Aufhebung der Kundschaften, Zeugnisse, Wanderpässe für Handwerksgesellen und Arbeiter; Einführung der Wanderbücher, in: Seiner k.k. Majestät Franz des Ersten politische Gesetze und Verordnungen für sämmtliche Provinzen des Österreichischen Kaiserstaates, mit Ausnahme von Ungarn und Siebenbürgen, Wien 1829, S. 231f; Kundmachung 231, in: Politische Gesetze und Verordnungen 1828, S. 283-289; Stopp 1982. 
Ungelernte, Jugendliche und Lehrlinge. ${ }^{6} 1902$ wurde diese Bestimmung auf Beschäftigte im Eisenbahnbau ausgedehnt. ${ }^{7}$ Analog dazu mussten häusliche Dienstbot*innen und ländliches Gesinde über Dienstbotenbücher verfügen (Morgenstern 1912, S. 120-128), Seemänner über Seedienstbücher (Verkauf 1905, S. 158f.). Diese Ausweise kategorisierten und unterschieden eine Arbeiterschaft. Höhere Angestellte benötigten keine solchen Bücher, sondern Zeugnisse in anderer Form. Arbeit- und Dienstgeber*innen mussten sich - zumindest gegenüber den Arbeiter*innen und Dienstbot"innen - nicht ausweisen (Ebenhoch/Pernersdorfer 1893, S. 154). Von den Bestimmungen ausgenommen blieben aber auch, trotz vermutlich häufig wechselnder Beschäftigung und Mobilität, die Lohnarbeiter*innen der "geringsten Art“, also Tagelöhner"innen und Gelegenheitsarbeiter"innen.

Von zeitgenössischen Kritiker*innen wurden die Dokumente deshalb als „äußerliches Zeichen“ einer Rechtsungleichheit (Arbeitsbücher 1912, S.13; Stenographische Protokolle 1913, S. 8050) betrachtet. Das Arbeitsbuch war, in den Worten sozialdemokratischer Abgeordneter, „das Merkmal der Sklaverei, der Hörigkeit, der gelbe Fleck, der allen denen angeheftet wird, die als Ausgestoßene, als Minderwertige, als Kontrollbedürftige angesehen werden. Es erregt von neuem immer wieder die Vorstellung von der Zweiteilung der Gesellschaft, in die Teilung von Kontrollbedürftigen und Kontrollosen." (Antrag des Abgeordneten Smitka, Palme und Genossen, 1911, S. 3; analog zum Dienstbotenbuch Popp, 1912, S. 12 und 31) Auch Historiker*innen haben diese Dokumente (vorwiegend) als Element der Disziplinierung und Unfreiheit von Arbeit (zur Diskussion vgl. Keiser 2013) sowie als sicherheitspolitische Maßnahme betrachtet. Die Politik gegenüber mobilen, als gefährlich geltenden und zugleich vulnerablen Bevölkerungsgruppen konterkarierte die Abschaffung der Inlandspässe und die Liberalisierung des internationalen Migrationsregimes im späten 19. Jahrhundert (vgl. Lucassen 2001). ${ }^{8}$

Arbeits- und Dienstbotenbücher setzten in dieser Hinsicht gleich, sie schufen ähnliche (in den Augen der Arbeiter*innen- und Dienstbot*innenbewegung ähnlich diskriminierende) Voraussetzungen für Arbeit und Mobilität. Gleichzeitig dokumentierten Arbeits- und Dienstbotenbücher aber

6 Arbeitsbücher für Minderjährige und Jugendliche waren noch am ehesten Konsens. Vgl. Sitzungs-Protokolle des ständigen Arbeitsbeitrates 1914, S. 94.

7 RGBl. 1860/66, RGBl. 1885/22, RGBl. 1902/156.

8 Keiser weist neben der disziplinierenden Funktion auch auf die Bedeutung hinsichtlich der Arbeitsmärkte hin. Keiser 2013, S. 391. 
auch einen formellen Status, ${ }^{9}$ der in vielerlei Hinsicht differierte und variierte, der mit unterschiedlichen Lebens- und Arbeitsverhältnissen, Sicherheiten und Gefahren und mit Unterschieden in Selbstsicht und Aspirationen verknüpft war. Anders als in der politischen Propaganda lässt sich unter Arbeiter*innen und Dienstbot*innen viel Abneigung gegen diese Dokumente, doch keineswegs eine einheitliche Haltung und Umgangsweise feststellen. Auch Arbeit-/Dienstgeber*innen und Behörden, die in ihren offiziellen Stellungnahmen auf Arbeits- und Dienstbotenbücher als unverzichtbares Instrument für die Aufrechterhaltung von Sicherheit und Ordnung bestanden (Ebenhoch/Pernersdorfer 1893, S. 416), agierten keineswegs geschlossen. Tatsächlich wurden die gesetzlichen Bestimmungen nur eher zögerlich durchgesetzt. Bis ins 20. Jahrhundert berichteten Zeitgenoss"innen vielfach von fehlenden Dokumenten (vgl. Bericht der k.k. Gewerbe-Inspectoren über ihre Amtsthätigkeit. Wien 1884-1916).

Alle Facetten des Problems der Sicherheit, der Arbeit, der sozialen Rechte, historischer Veränderungen und regionaler Unterschiede innerhalb der im Reichsrat vertretenen Königreiche und Länder der Habsburgermonarchie erschöpfend und im Detail zu behandeln, würde den Rahmen dieses Beitrags sprengen. Im Folgenden skizziere ich in nur sehr groben Zügen, wie sich die mit Ausweisdokumenten verknüpften Gefahren und Sicherheiten in der Argumentation der involvierten Parteien darstellten, wie sie in verschiedenen Kontexten zur Herstellung von Unterschieden benutzt werden konnten. Ich beziehe mich dabei auf politische Schriften, Zeitungsberichte, Rechtsdarstellungen und Autobiografien sowie Verwaltungsmaterialien. Ich werde zunächst Ähnlichkeiten und Verschiedenheit von Arbeits- und Dienstbotenbüchern in Hinblick auf die vorgeschriebenen administrativen Verfahren, die Gestaltung der Dokumente und die damit verknüptten Sicherheitsargumente und -erwartungen beschreiben. Im zweiten Teil des Textes erläutere ich die verschiedenen Möglichkeiten, die Dokumente zum Herstellen und/oder Unterlaufen von Unterschieden zu nutzen. Ich konzentriere mich dabei auf Behörden, Arbeiter*innen/Dienstbot"innen, Arbeit-/Dienstgeber*innen und lasse andere Parteien und Kontexte der Benutzung solcher Dokumente weitgehend außen vor.

9 Sie unterlagen beide der Verrechtlichung und Formalisierung, was aber in der Arbeitsrechtsgeschichte sehr unterschiedlich wahrgenommen wurde. Vgl. etwa Pierson 2016 und Vormbaum 1980, S. 134ff. 


\section{Die Sicherheit der Dokumente}

Identitäts- und Reisedokumente (vgl. Groebner 2004) sowie die Forderung, dass Arbeiter*innen und Dienstbot*innen mit Papieren ausgestattet sein sollten, die ihr reguläres Ausscheiden aus einer Stelle belegten und die zugleich die Voraussetzung für eine Anstellung sein sollten, waren keine Erfindung des 19. Jahrhunderts. Schon in der Frühen Neuzeit finden sich vielfach Erlässe und Vorschriften zu Pässen, Entlassungspapieren oder Zeugnissen für Handwerker, Gesinde und Dienstbot*innen (vgl. Morgenstern 1902, S. 22 ff.). Allerdings scheinen diese Erlässe nicht per se wirksam gewesen zu sein, es wurden häufig fehlende Dokumente, Missbräuche und Fälschungen beklagt. Darauf bezogen sich auch die Reformen des 19. Jahrhunderts, mit denen Ausweispapiere und ihre Ausstellung zunehmend reguliert, vereinheitlicht, kontrolliert und zur Sache der staatlichen Behörden gemacht wurden (vgl. Heindl/Saurer 2000). Im Großen und Ganzen entspricht dies der von Torpey beschriebenen Tendenz zur Monopolisierung der legitimen Mittel des Reisens (vgl. Torpey 2000). Dabei handelt es sich allerdings nicht um eine lineare, problem- oder konfliktfreie Entwicklung (vgl. dazu auch Higgs 2011). Es finden sich im Laufe des 19. Jahrhunderts immer wieder Verordnungen, die den Verkauf von sog. ,Blanquetten $^{6}$ (Vordrucken) untersagten. ${ }^{10}$ Genossenschaften, Arbeit-/Dienstgeber*innen und andere Parteien blieben in die Produktion und die Kontrolle dieser Dokumente involviert.

Arbeits- und Dienstbotenbücher waren äußerlich, in Hinblick auf Form und Format recht ähnlich; sie differierten in Hinblick auf die gesetzlichen Grundlagen, das Verfahren, die involvierten Behörden und die erfassten Daten. ${ }^{11}$ Die Bestimmungen hinsichtlich der Arbeitsbücher waren Teil der Gewerbeordnung und ihrer Novellen, die für alle im Reichsrat vertretenen Königreiche und Länder galten. Dienstbotenbücher waren auf der Ebene der Länder oder Städte in den Dienstboten- und Gesindeordnungen geregelt, es gab also keine einheitlichen Bestimmungen (vgl. Morgenstern 1902 und 1912). Die Ausstellung der Dokumente lag in den Händen der Ge-

10 Z. B. LGBl. Steiermark 1871/36, 1897/79; LGBl. Oberösterreich 1871/15, LGBl. Kärnten 1879/14.

11 Arbeits- und Dienstbotenbücher sind bislang wenig erforscht und wurden kaum je systematisch verglichen. In Hinblick auf die Rechtsgeschichte diskutieren etwa Pierson 2016 und Vormbaum 2014 die Verrechtlichung des Dienstes im Vergleich mit Lohnarbeit. 
meindeverwaltung ${ }^{12}$, im Fall der Arbeitsbücher bei der Aufenthaltsgemeinde, im Fall der Dienstbotenbücher prinzipiell bei der nach dem Heimatrecht zuständigen Heimatgemeinde ${ }^{13}$ (vgl. Morgenstern, 1912, S. 121; Richter 2017, S. 52-63). Die Ausfertigung, die Angaben zur Person und die zugrundeliegenden Dokumente wurden in Arbeits- und Dienstbotenbuchprotokollen registriert.

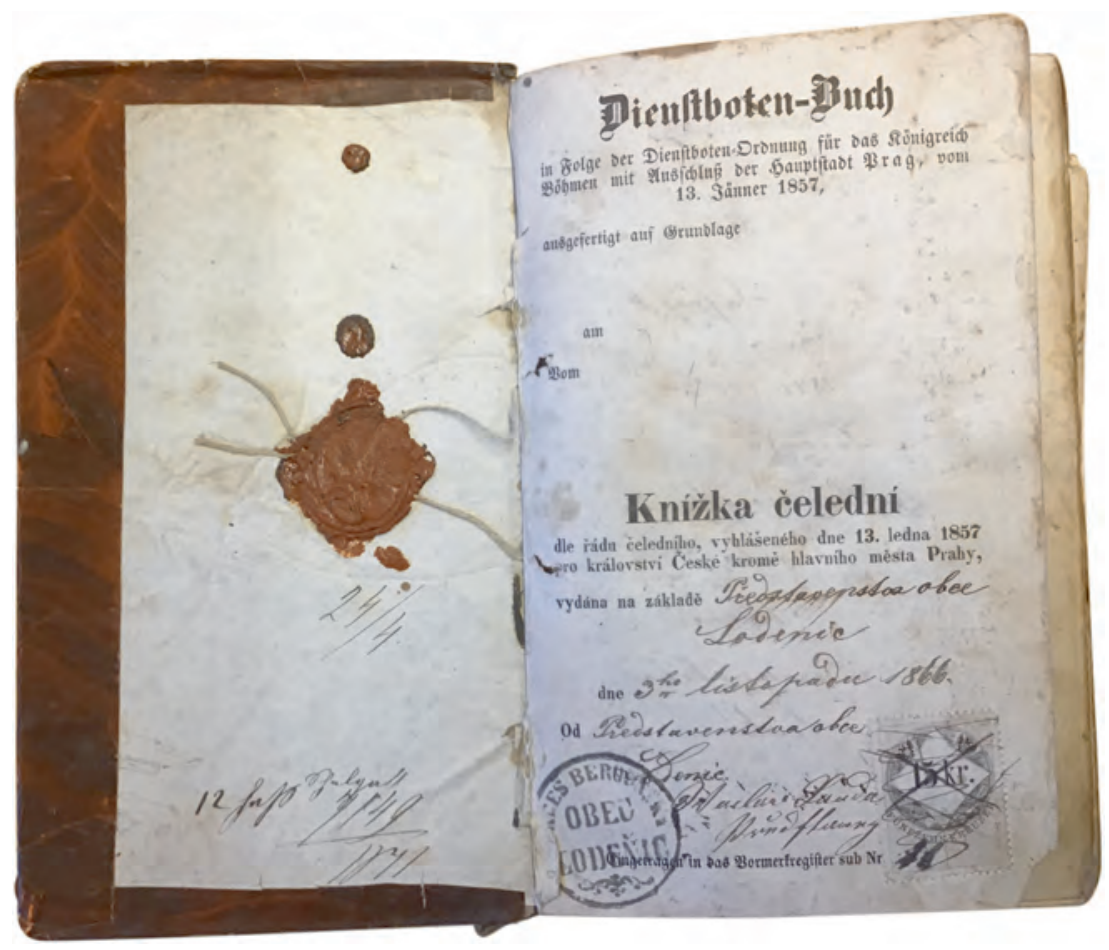

Abb. 1: WStLA, Versorgungshaus Mauerbach, A6-Arbeitsbücher-Muster, Schachtel 2: Dienstbotenbuch Anna Šafárík

12 Zur Stellung der Gemeinden in der Verfassung und Verwaltung der Habsburgermonarchie vgl. Deak 2015, S. 151f.

13 Mit Zustimmung der Heimatgemeinde konnte auch die Gesindebehörde der Aufenthaltsgemeinde ein Dokument ausstellen. 


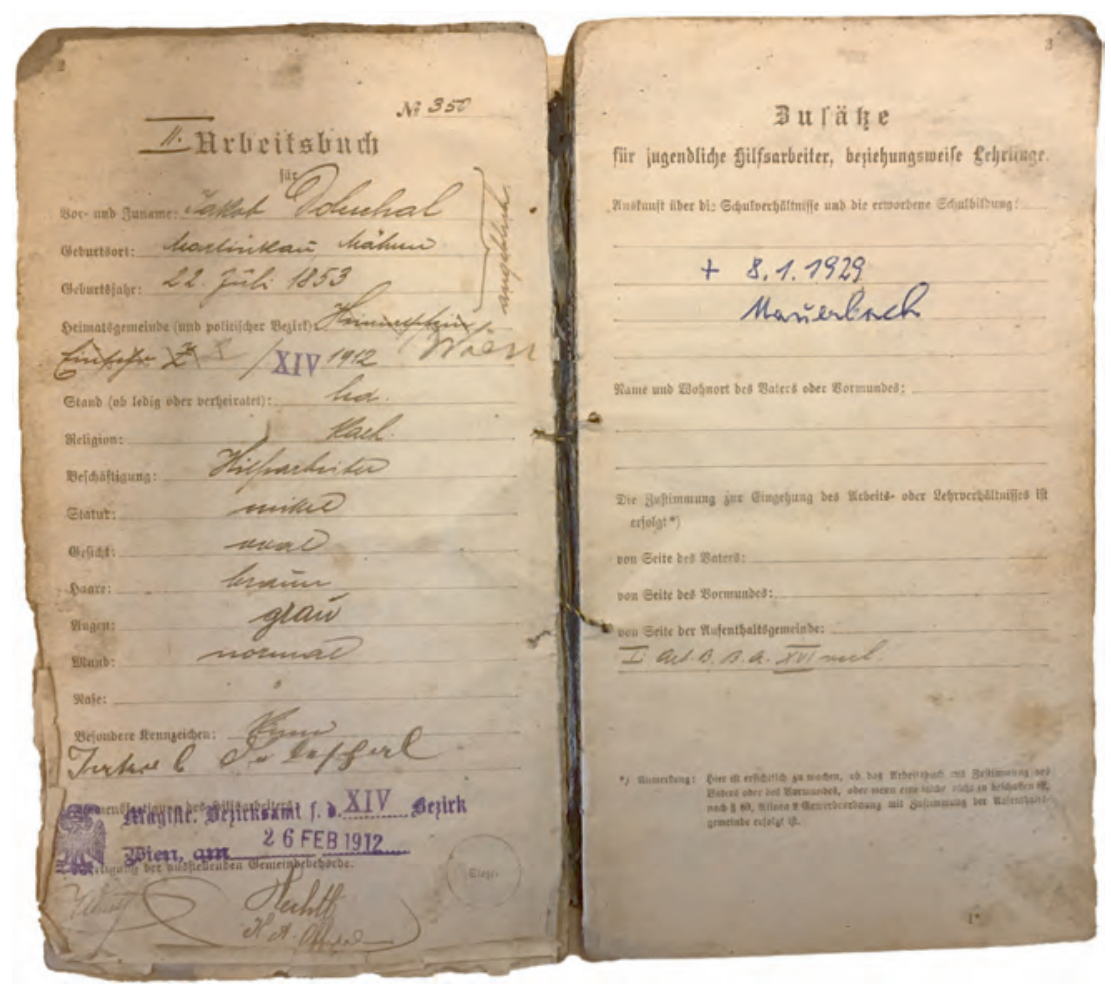

Abb. 2: WStLA, Versorgungshaus Mauerbach, A6-Arbeitsbücher-Muster, Schachtel 2: Arbeitsbuch Jakob Doleschal

Die Büchlein (vgl. Abb. 1 und 2) waren, vergleicht man sie mit anderen zeitgenössischen Identitätsdokumenten, umfangreich und dabei klein, handlich und strapazierfähig gestaltet, meist im Oktave-Format oder ähnlichem. Die Seiten waren durch einen Faden verbunden, der durch ein Siegel der ausstellenden Behörde fixiert war. Damit sollte das Entfernen einzelner Seiten verhindert werden. In Arbeitsbüchern war ab 1885 auf der ersten Seite als weiteres Sicherheitsmerkmal auch die Anzahl der Blätter und ein Inhaltsverzeichnis mit Seitenangaben abgedruckt. Die konkrete Gestaltung der Formulare, die Anordnung, die Genauigkeit der Einträge variierte und veränderte sich im Laufe der Untersuchungsperiode. Es gab 
regionale Unterschiede, wie etwa ein- oder zweisprachige Formulare. ${ }^{14}$ Auf den ersten Seiten fanden sich Angaben zur Art des Dokuments, der ausstellenden Behörde, das Datum der Ausstellung und die Nummer, die auf das Arbeits- und Dienstbotenbuchprotokoll der Gemeinde verwies. Es folgten die Personalien (in mehr oder minder großer Genauigkeit und Detailliertheit), die Personenbeschreibung, die Unterschrift des Inhabers/der Inhaberin (so er/sie schreiben konnte) sowie die Fertigung der ausstellenden Gemeindebehörde. Im Anschluss konnten Reisebewilligungen eingetragen werden, diese galten zeitlich befristet für die angeführten Länder (RGBl. 1860/66). Ein größerer Anteil an Seiten war für die fortlaufende Dokumentation der Arbeits- oder Dienstverhältnisse vorgesehen: Angaben über Dienst- oder Arbeitgeber*in (Gewerbe, Standort des Betriebs), Datum des Ein- und Austrittes sowie Arbeitszeugnisse in verschiedener Form. Im Fall von Arbeitsbüchern fanden sich hier handschriftliche, meist formelhafte Einträge der Arbeitgeber*innen oder auch gestempelte Zeugnisse mit Raum für handschriftliche Ergänzungen. Dienstbotenbücher wiesen häufig vorgedruckte Spalten auf, in denen Treue, Geschicklichkeit, Fleiß und Sittlichkeit des Inhabers/der Inhaberin zu bestätigen waren. Die Dienstzeugnisse wurden - anders als die Zeugnisse in Arbeitsbüchern, wo dies möglich, aber nicht verpflichtend war - generell von den Behörden auf der Grundlage der Angaben der Dienstgeber*innen eingetragen ${ }^{15}$ (vgl. Morgenstern 1912, S. 124). Einträge der Arbeits- oder Dienstgeber“innen waren - zumindest war dies so vorgeschrieben - von der Gemeinde, von der Genossenschaft oder der örtlichen Polizei zu bestätigen. Die Ausstellung und Benutzung der Dokumente verlangten also regelmäßige Interaktionen mit Behörden, diese sollten routinemäßig in die Begründung und Aufösung von Arbeitsverhältnissen involviert sein. Die Bestimmungen der Gesinde- oder Gewerbeordnung waren auszugsweise in den Büchern abgedruckt. Ab 1885 sahen die Formulare zusätzliche Angaben zur Schulbildung, zu Vater oder Vormund vor, dessen Zustimmung zum Arbeits- oder Lehrverhältnis war zu vermerken. (RGBl. 1885/69) In Arbeitsbüchern für Lehrlinge waren die wichtigsten Bedingungen des Lehrvertrages anzugeben. In manchen Arbeitsbüchern gab es darüber hinaus noch weitere Einträge, etwa Stempel von Naturalverpflegsstationen (Herbergen), Bahnstationen, Bestätigungen über Krankenhausaufenthalte. Dienstbotenbücher

14 Beispiele finden sich in vielen Archiven, im Wiener Stadt- und Landesarchiv (WStLA) etwa in der Dokumentensammlung oder den Beständen des Versorgungshauses Mauerbach.

15 Nur in Tirol schrieben sie Dienstgeber*innen eigenhändig in die Dienstbotenbücher ein. 
beinhalteten Angaben zum Angeld (ein Vorschuss, mit dem der Abschluss des Arbeitsvertrages verbindlich wurde), zum vereinbarten Jahreslohn und der Dauer des Vertrages. Manchmal wurde auch festgehalten, wo die Zeit zwischen zwei Dienstverhältnissen verbracht wurde, etwa ob sich jemand bei ihrer/seiner Familie aufgehalten hatte. Die Dokumente verbrieften also nicht nur den Beruf, Ausbildung bzw. die Art des Erwerbs, grundlegende rechtliche Bedingungen der Arbeits- bzw. Dienstverhältnisse und konkrete Übereinkünfte, sie sollten - das war ein wesentlicher Kritikpunkt - wie ein „Steckbrief“ über einen längeren Zeitraum Verhalten, Arbeit/Dienst und auch Erwerbslosigkeit dokumentieren (vgl. Ingwer 1895, S. 24; Ebenhoch/ Pernersdorfer 1893, S. 86 und 211; Verkauf 1905, S. 158; Popp 1912, S. 31 ).

\section{Die Sicherheit der Benutzer*innen der Dokumente}

In den Auseinandersetzungen über die Dokumente insistierten Behörden, Arbeit- und Dienstgeber*innen, dass diese in genau dieser Form zur Aufrechterhaltung von Ordnung (vgl. Ebenhoch/Pernersdorfer 1893, S. 154) bzw. häuslicher Ordnung (vgl. Richter 2017, S. 56) und Sicherheit unverzichtbar wären. Aber was genau war damit gemeint? Welche Ordnung? Welche Sicherheit? Wessen Sicherheit? Die Dokumente wurden - wie bereits in den Verordnungen des 18. Jahrhunderts argumentiert - als Mittel zur Bekämpfung von Vagabundage und Müßiggang betrachtet. Aus behördlicher Perspektive unzuverlässigen oder vermeintlich arbeitsscheuen Individuen sollten keine Arbeits- und Dienstbotenbücher ausgestellt werden (vgl. Erlass der k.k. Statthalterei für Oberösterreich, 1865), beziehungsweise sollten diese durch die Dokumente kenntlich gemacht werden (vgl. Mayerhofer 1897, S. 543). Jemanden zu beschäftigen, der keine Dokumente besaß oder sein Arbeitsverhältnis zuvor nicht ordnungsgemäß beendet hatte, war untersagt. Damit sollten das „Entlaufen“ (die vertragswidrige, vorzeitige Beendigung des Arbeitsverhältnisses individuell oder auf Grund eines Streiks), aber auch allzu häufiger Stellenwechsel, Missbrauch mit Lohnvorschüssen ${ }^{16}$, das Abwerben fremder Arbeiter*innen/Dienstbot*innen unterbunden und Löhne reguliert werden. Die Dokumente sollten Arbeit- und Dienstgeber*innen in die Lage versetzen, die Identität der Person sicherzustellen und sich vorab der Eignung, Erfahrung, des Flei-

16 Etwa einen Vorschuss anzunehmen, ohne dann auch die Arbeit anzutreten. 
ßes, der Treue und Zuverlässigkeit einer Person zu vergewissern. ${ }^{17}$ Konnte man der Person, die man in Arbeit oder in Dienst (und in diesem Fall auch in den Haushalt auf-) nahm, Vertrauen entgegenbringen (vgl. Sozialpolitischer Ausschuss, 1913, S. 3)? Arbeits- und Dienstbotenbücher mussten bei Antritt einer Stelle dem/der Arbeit-/Dienstgeber*in ausgehändigt werden, sie verblieben während der Dauer des Beschäftigungsverhältnisses - quasi als Pfand - dort. Erst bei Beendigung des Arbeitsverhältnisses sollten sie, mit einem Zeugnis versehen, wieder dem/der Arbeiter"in/Dienstbot"in ausgehändigt werden. Dienstgeber*innen waren verpflichtet, die Dienstbot"innen, die ja im Haushalt des Arbeitgebers wohnten, polizeilich zu melden. Es gab eigene Meldezettel für weibliche und männliche Dienstbot"innen. In größeren Gewerbe- und Industriebetrieben waren Arbeitgeber*innen verpflichtet, Register ihrer Arbeiter*innen zu führen (RGBl. 1859/227, \83). In Hinblick auf ihre Unterkunft unterlagen diese, wie die anwesende Bevölkerung generell, dem polizeilichen Meldewesen (vgl. Polizeidirektion 1920; Ehrenfreund 1908, S. 8). Dokumente und Register verwiesen also aufeinander.

In dieser Perspektive dienten Arbeits- und Dienstbotenbücher und die damit verknüpften Registrierungs- und Meldepflichten der behördlichen Überwachung und Evidenz (vgl. Ehrenfreund 1908, S. 33), der Sicherheit der Arbeit- und Dienstgeber*innen, ihrer Betriebe (z.B. Die Abschaffung des Arbeitsbuches auf Grund des $\mathbb{1} 14$ 1914, S. 17) und Haushalte, sie wurden als „Compell“ zur Einhaltung der Arbeitsverträge betrachtet (Ingwer 1895, S. 17). Diese Aspekte überwogen zweifellos in den Debatten. In Hinblick auf Arbeitsbücher spielten Streik und politische Agitation eine größere Rolle. In Hinblick auf die Dienstbotenbücher kamen zu Gefahren bzw. Sicherheiten für Ansprüche, Eigentum und Leben der Dienstgeber*innen noch häufig solche der Moral und der Sittlichkeit hinzu. Dies galt für die Familie des Dienstgebers/der Dienstgeberin, aber freilich waren auch Dienstbot*innen, vor allem Dienstmädchen, Gefahren ausgesetzt („,Wer bürgt den Dienstboten für ihre persönliche Sicherheit angesichts

17 „Sowohl vom gewerblichen als vom sozialen Standpunkte aus ist die Aufhebung der Arbeitsbücher sehr bedauerlich, denn in den Arbeitsbüchern ersah man ziemlich deutlich, ob der Arbeiter ein ordentlicher Mensch war, und es ist wohl nicht gleichgültig, ohne irgendwelche Sicherheit einem Menschen, den man sonst nicht kennt, Vermögen und die Sicherheit der Gesundheit der Mitmenschen anzuvertrauen. Hatte man in dem Arbeitsbuche gesehen, daß der Arbeiter lange dauernde Arbeitsplätze hatte, so wußte man auch, daß er ein verläßlicher Mensch ist." Die Aufhebung der Arbeitsbücher 1919, S. 7. 
des in puncto Moral sehr anrüchigen Vorlebens der Ehegatten verschiedener ,Gnädigen?"“ Vermischtes 1893, S. 8; vgl. dazu auch Richter 2017).

Befürworter der Ausweisdokumente führten ins Treffen, dass diese Dokumente auch für Arbeiter*innen und Dienstbot"innen von Nutzen sein konnten. Arbeits- und Dienstbotenbücher würden in praktischer Weise alle notwendigen Dokumente bündeln, ungehindertes Reisen und Arbeitsuchen, also Freizügigkeit ermöglichen. Sie sollten (wandernde) Arbeitsuchende von - vermeintlich - arbeitsscheuen Vagabunden unterscheiden und damit auch redliche Arbeiter*innen/Dienstbot*innen vor Verdächtigungen und dem Zugriff der Polizei bewahren, also vor missbräuchlichen Anwendungen des Vagabundengesetzes schützen. Sie boten Zugang zu Bahnermäßigungen oder zu Naturalverpflegsstationen für arbeitssuchende Wanderer, die allerdings in der Monarchie primär männlichen Gesellen und Facharbeitern offenstanden (vgl. Wadauer 2015). Dokumente ermöglichten es, die Zugehörigkeit zu einer Branche nachzuweisen und Ansprüche zu stellen (vgl. Ebenhoch/Pernersdorfer 1893, S. 153). Darüber hinaus stellten Belege beruflicher Qualifikation und Erfahrung die Voraussetzung für Fortkommen und berufliche Laufbahn dar, der Nachweis der Gesellenarbeit war für die Erlangung des Meisterrechts erforderlich. „Das Arbeitsbuch liefert für den anständigen Arbeiter ein förmliches Lebensbild, es enthält eine förmliche Biographie desselben von dem Augenblicke an, wo er in die Lehre tritt." (Morgenstern 1912, S. 126). ${ }^{18}$ Ein tüchtiger und fleiBiger Mensch könnte stolz auf seine Dokumente sein, diese könnten ihm nicht zum Nachteil gereichen (vgl. Ebenhoch/Pernersdorfer 1893, S. 209f.). Ein Zeugnis zu erhalten, war auch ein Recht (vgl. Sozialpolitischer Ausschuss 1913, S. 4). Dass diese Dokumente im Prinzip allen Beteiligten - zumindest den anständigen und tüchtigen - gleichermaßen Vorteile und Sicherheit(en) bieten konnten, war allerdings nicht Konsens. Jeder der hier angesprochenen Punkte warf Fragen auf, erforderte Interpretation und Grenzziehungen, die je nach Interesse, Standpunkt und Situation oft divergierten.

Am erstaunlichsten war, dass die Umsetzung dieser Politik an den Behörden selbst scheiterte, die weder einheitlich noch immer im Sinn der Gesetze agierten. Auf der Ebene der Gemeindeverwaltung mangelte es den Berichten der Gewerbeinspektoren zufolge - oft an Verwaltungskompetenz und Rechtskundigkeit, man war mit den Bestimmungen nicht ver-

18 Analog dazu wurde mit dem Dienstbotenbuch angestrebt, „einen zusammenhängenden, verläßlichen, behördlich bestätigten Ausweis über Dienstleistung und Verhalten der Dienstboten während der ganzen Dienstzeit zu erlangen.“ Ebd. 
traut, manchmal fehlten schlicht die gültigen Formulare. Auch die Zuständigkeit war oft unklar und strittig, etwa was als Aufenthaltsgemeinde zu verstehen war, aber auch wem überhaupt welche Dokumente auszustellen waren. In Hinblick auf Staatsbürgerschaft war kein Unterschied zu machen, so die Person nicht bereits entsprechende Dokumente des Heimatlandes besaß. Manche Gemeinden weigerten sich aber, Arbeitsbücher an Nicht-Heimatberechtigte auszustellen, da sie fürchteten, dass damit auch Ansprüche an die Gemeinde begründet wären. Andere Gemeinden hingegen betrachteten die Ausstellung der Dokumente an Einheimische oder an Jugendliche als überflüssig (vgl. z.B. Bericht der k.k. Gewerbe-Inspectoren z.B. 1885, S. 71; 1899, S. LXVII; 1903, S. 113; 1909, S. CXXI; 1911, S. CLVIf etc.).

Auch die Unterscheidung zwischen Erwerbstätigkeiten, die ein Arbeitsbuch erforderten und solchen, die ein Dienstbuch verlangten, also der Gewerbe- oder der jeweils geltenden Gesindeordnung unterlagen, war keineswegs klar und unstrittig (vgl. Ehrenfreund 1908, S. 34-36; Richter 2017, S. 204-286). Gemeinden orientierten sich bei der Ausfertigung der Dokumente, wie von den Gewerbeinspektoren bemängelt wurde, oft am Geschlecht der Person, nicht am Arbeitsverhältnis oder dem Arbeitsplatz (z.B. Bericht der k.k. Gewerbe-Inspectoren 1907, S. CXXXIII). Tatsächlich übten ja nicht nur, aber zum größeren Teil Männer diejenigen Berufe aus, die Arbeitsbücher erforderten. Diejenigen Erwerbe, die ein Dienstbotenbuch erforderten, wurden im 19. Jahrhundert vor allem und immer mehr von Frauen ausgeübt, besonders deutlich ist dies im häuslichen Dienst, in dem gegen Ende des 19. Jahrhunderts fast ausschließlich Frauen beschäftigt waren (vgl. Morgenstern 1902, Kapitel 6; Winter 1900, S. 585; Volkszählung 1910, S. 11 und 64). Die historische Forschung spricht von einer „Feminisierung“ des (häuslichen) Dienstes im Laufe des 19. Jahrhunderts (Richter 2017, S. 20; Hoerder 2015; Sarti 2014, S. 293). Allerdings konnten manche Tätigkeiten als Dienst wie auch als gewerbliche Hilfsarbeit ausgeübt werden. Als Koch/Köchin konnte man in einem Gewerbebetrieb oder im Privathaushalt beschäftigt sein, Wäschewaschen war Aufgabe häuslicher Dienstbot*innen und Geschäft der gewerblichen Wäschereien. Die Grenze zwischen Gewerbebetrieb und Haushalt war im Einzelnen nicht immer einfach zu ziehen. Was war überhaupt unter einem Haushalt, einem Betrieb oder einer Landwirtschaft zu verstehen? Auch in Gewerbebetrieben gab es zumindest bis zur Gewerbeordnungsnovelle 1885 Dienstbot"innen, auch in Anstalten gab es Hauspersonal, auch in Haushalten gewerbliche Arbeiter"innen (vgl. dazu ausführlich Richter 2017, S. 204-286). Dienstbote/Dienstbotin oder Arbeiter"in zu sein, war zudem nicht eine fixe Eigenschaft einer Person, viele übten im Laufe ihres Lebens verschiede- 
ne wechselnde Erwerbe, oft auch mehrere Erwerbe gleichzeitig aus (vgl. Bericht der k.k. Gewerbe-Inspectoren 1894, S. 18; Ehrenfreund 1908, S. 35).

Die Kategorisierung der Tätigkeiten, die Ausstellung der entsprechenden Dokumente war strittig, weil dies auf relevante Unterschiede und auf Hierarchien von Arbeit bzw. Lebensunterhalten verwies. Handwerker, die sich als Künstler verstanden, Kleinbauern, die nur saisonal Lohnarbeit verrichteten, sahen sich ungerechtfertigt mit Arbeiter*innen und Dienstbot"innen auf eine Ebene gestellt und damit herabgesetzt (vgl. Ertl 1886, S. 56; Bericht 1886, S. 22). Häufig findet sich in Publikationen der Arbeiterbewegung das Argument, dass nur „Arbeiter und Dirnen“ solche entwürdigenden Dokumente besitzen mussten (Ingwer, 1912, S. 14). Der mit den Dokumenten verbriefte Status als Arbeiter*in- und Dienstbot*in hatte auch handfeste Konsequenzen berufs- und sozialrechtlicher Natur, war mit unterschiedlichen, zunehmend formalisierten Rechten und Pflichten verknüpft. Es gab eine Diskrepanz in den Gesetzen und Arbeitsschutzbestimmungen. ${ }^{19}$ Die Kranken- und Unfallversicherung für gewerbliche Hilfsarbeiter*innen etwa wurde bereits in den 1880er Jahren eingeführt (vgl. Tálos/Wörister 1994, S. 17). Für Dienstbot*innen galt bis zu deren Einbezug in die Arbeiterkrankenversicherung 1922 die Fürsorgepflicht des Dienstgebers/der Dienstgeberin. Etwaige Versicherungen zielten lediglich darauf ab, letztere vor finanziellem Schaden zu bewahren. Die Arbeitsbedingungen und Arbeitsordnungen in Gewerbe und Industrie unterlagen prinzipiell - der Kontrolle der 1883 eingerichteten Gewerbeinspektorate (RGBl. 1883/117). Im Konfliktfall und bei Beschwerden, bei Verstößen gegen die Schutzbestimmungen und Arbeitsordnungen waren Schiedsgerichte der Genossenschaften und Gewerbegerichte (RGBl. 1869/63) zuständig. Die Beschäftigung im Haushalt unterlag hingegen keiner Aufsicht, im Fall der Dienstbotenstreitigkeiten waren Polizei oder Bürgermeister (vgl.

19 Es findet sich auch eine ungleiche Präsenz dieser Fragen in der zeitgenössischen Presse. In den digitalisierten Zeitschriftenbeständen der österreichischen Nationalbibliothek (http://anno.onb.ac.at/) sind hunderte Artikel betreffend den Schutz und Sicherheit von Arbeiter*innen in ihrer Arbeit zu finden, kaum jedoch solche zu Schutz und Sicherheit von Dienstbot*innen/Dienstmädchen/des Gesindes, zumindest, wenn man nach diesen Wortkombinationen sucht. Nicht zuletzt trug die Presse der organisierten Arbeiterbewegung dazu bei, solche Themen in die Zeitungs-Öffentlichkeit zu bringen. Gewichtungen in den digitalisierten Beständen sind dabei in Erwägung zu ziehen, allerdings sind frauenspezifische Publikationen ein Schwerpunkt der digitalen Sammlung der Nationalbibliothek. 
Richter 2017, S. 53$)^{20}$ die Adressaten. Verstöße gegen die Bestimmungen sowie der Bruch des Arbeitsvertrages zogen unterschiedliche Konsequenzen nach sich. Vertragsbrüchigen gewerblichen Hilfsarbeiter"innen drohte Arrest, Schadenersatz und eine zwangsweise Rückführung an den Arbeitsplatz (vgl. Heilinger 1909, S. 541 und 545). Dienstbot*innen, die vorzeitig ohne gesetzlich vorgesehenen Grund ihre Arbeit verließen und ihren Lohnvertrag verletzten, mussten bis ins 20. Jahrhundert im Extremfall mit körperlicher Züchtigung und Rückführung an den Arbeitsplatz rechnen (vgl. Morgenstern 1912, S. 133; Althaus 2010, S. 283; Verkauf 1905, S. 178 182). Dienstgeber"innen hingegen konnten im Streitfall zu Schadenersatz oder Geldstrafen verurteilt werden, gegebenenfalls konnte ihnen das Recht entzogen werden, Lehrlinge auszubilden (vgl. Ingwer 1895, S.9; RGBl. $1859 / 227, \mathbb{1 3 7}$ ).

Manche Arbeitgeber*innen machten sich solche Unterschiede im Arbeitsrecht zu nutze. Jahr für Jahr bemängelten Gewerbeinspektoren, dass Betriebe Arbeiterinnen als Dienstbotinnen oder Arbeiter*innen nur als Arbeiter*innen der geringsten Art - also unter schlechteren arbeits- und sozialrechtlichen Bedingungen, ohne Versicherungsanspruch - beschäftigten (Bericht der k.k. Gewerbe-Inspectoren 1886, S. 122 oder 1913, S. CLXXVf.). Jahr für Jahr wurde auch berichtet, dass Arbeitgeber*innen, die im politischen Diskurs so vehement auf der Unverzichtbarkeit der Dokumente beharrten, Arbeiter*innen ganz ohne die vorgeschriebenen Arbeitsdokumente einstellten. Dies war in manchen Regionen mit vielen ausländischen Arbeitskräften, in Branchen mit starker Fluktuation und stark wechselndem Arbeitskraftbedarf besonders oft der Fall. Auch in Hinblick auf Dienstbot*innen, vor allem aber ländliches Gesinde wurde Ähnliches berichtet. Bei solchen ohne die vorgeschriebenen Dokumente Beschäftigten musste es sich freilich nicht um völlig unbekannte Personen ohne jeglichen Identitätsausweis und ohne jegliche Referenz handeln. „Ich kenne meine Leute", rechtfertigte etwa ein Kleingewerbetreibender seine Missachtung staatlicher Bestimmungen (Bericht der k.k. Gewerbe-Inspectoren 1889, S. 85). Mit der Formalisierung und Registrierungspflicht von Arbeit, mit der Entwicklung sozialer Sicherungssysteme entstand langfristig ein neuer Gegensatz, der seit den 1970ern als Gegensatz von „formeller“ und „informeller“ Arbeit (Hart 1973) diskutiert wird.

Der Durchsetzung der Ausweisdokumente wirkte wohl auch entgegen, dass vielfach bezweifelt wurde, ob diese überhaupt aussagekräftig waren

20 Ein Gericht war nur dann zuständig, wenn das Dienstverhältnis nicht mehr aufrecht war. Richter 2017, S. 53. 
und ein Urteil über eine Person erlaubten (Sozialpolitischer Ausschuss 1913, S. 3). Ein zentraler Streitpunkt waren die Arbeitszeugnisse, die wahrheitsgemäß ausgestellt werden mussten, aber auch nicht explizit negativ sein durften; sie sollten niemanden in seinem/ihrem Erwerb behindern. Der Schutz der Arbeiter*innen und Dienstbot"innen stand hier deutlich dem Schutz der Arbeit- oder Dienstgeber"innen gegenüber (vgl. etwa Die Dienstbotenfrage in Wien 1892, S.9-11). Manche Arbeit-/Dienstgeber*innen gaben deshalb an, den Zeugnissen ohnehin keinen Glauben zu schenken und - so sie überhaupt auf diesen Dokumenten beharrten - lediglich auf die Dauer der Anstellungen und die Häufigkeit des Arbeitsplatzwechsels zu achten. Es wurde aber auch vielfach gegen die Bestimmungen verstoßen. Manche Dienstgeber*innen trugen falsche positive Zeugnisse (vgl. Unsere Dienstboten-Misère 1876; Treu, fleißig, sittlich 1879), andere trotz allem negative Zeugnisse ein. Es reichte allerdings schon, Rubriken des Vordrucks im Dienstbotenbuch freizulassen, um zukünftige Dienstgeber*innen zu warnen (vgl. etwa die Erinnerungen von Weiss 1907, S. 233). Arbeitgeber*innen in Gewerbe und Industrie wurden in den 1890er Jahren beschuldigt, 'schwarze Listen' über organisierte und streikende Arbeiter*innen zu führen und Arbeiter*innen mit geheimen Zeichen oder mit bestimmten Formulierungen in den Zeugnissen als unzuverlässig, diebisch, faul, unfähig, renitent, unverträglich, als Trinker*in oder - etwa auch durch die Verwendung von Rot als Stempelfarbe - als Sozialist"in zu brandmarken (vgl. Ingwer 1895, S. 26; Ebenhoch/Pernersdorfer 1893, S. 255). Arbeitsbücher wurden also auch instrumentalisiert um gegen organisierte Arbeiter*innen vorzugehen und deren politische Rechte zu unterlaufen. Die Dokumente wurde in diesem Sinn sogar als ,Uriasbrief ${ }^{21}$ bezeichnet, sie überantworteten, so lautete die Kritik, die Existenz der Arbeiter"innen und Dienstbot"innen und ihrer Familien der Willkür und den Launen der Arbeitgeber*innen. Das Misstrauen war groß, die Arbeits-/Dienstzeugnisse waren häufig Gegenstand von Konflikten, Rechtsstreitigkeiten und Beschwerden, die auf Grund der Rechtsverhältnisse und Zuständigkeiten in Hinblick auf Arbeitsbücher eher dokumentiert und statistisch fassbar sind (z.B. durch die Sammlungen der Entscheidungen der k.k. Gewerbegerichte. Wien 1900-1920) als solche, die Dienstbotenbücher betrafen. Konnte ein schädlicher Eintrag nachgewiesen wer-

21 Der/die Überbringer*in übergab mit dem Dokument also quasi selbst sein/ihr eigenes Todesurteil oder zumindest einen Grund ihn/sie nicht anzustellen. Es handelt sich um eine Anspielung auf eine Geschichte aus dem Alten Testament (2 Sam 11). 
den, oder hielt ein/e Arbeitgeber"in ein Dokument ungerechtfertigt bei einer Kündigung zurück, so waren die Arbeitgeber*innen prinzipiell verpflichtet, Schadenersatz zu leisten und die Beschaffungskosten eines neuen Buches zu tragen.

Formell Beschwerde über ein Zeugnis oder die Nicht-Ausfolgung der Dokumente einzulegen, war allerdings nur eine, zudem eine zeitaufwändige Möglichkeit, mit diesen „Fesseln“ (Ingwer 1912, S. 14), umzugehen. Überaus häufig ließen Besitzer“innen ihre Dokumente zurück oder zerstörten sie einfach. In Fabriken, bei Ämtern, Arbeits- oder Dienstvermittler*innen wurden oft Hunderte solch zurückgelassener, beschädigter Dokumente aufbewahrt, da man nicht wusste, wie man damit verfahren sollte (vgl. Morgenstern 1912, S. 127; Heller 1907, S. 618; Bericht der k.k. Gewerbe-Inspectoren 1887, S. 22). Das ist insofern bemerkenswert, da Ausweislosigkeit ein Risiko darstellte und im Extremfall zu langer Haft zur Identitätsfeststellung führen konnte (vgl. Ingwer 1895, S. 26). Trotz aller Sicherheitsbestimmungen war es aber offensichtlich nicht allzu schwierig, sich ein Duplikat oder gar Triplikat zu besorgen (vgl. Ehrenfreund 1908, S. 37f.; Mayerhofer 1897, S. 543). Der schriftliche Nachweis beruflicher Erfahrung ging dabei zwar verloren, so die Zeugnisse nicht auch in anderer Form vorlagen, war jedoch offenkundig nicht in jeder Art der Erwerbstätigkeit gleichermaßen von Bedeutung. Nicht alle Arbeit-/Dienstgeber"innen konnten oder wollten schriftlichen Dokumenten denselben Wert zuweisen und sie genau prüfen.

\section{Gefahren und (offiziell) falsche Sicherheiten}

Aussagekraft und Zuverlässigkeit der Dokumente waren also im Einzelnen nicht leicht festzustellen, sie waren durchaus verhandelbar. $\mathrm{Zu}$ den Schwierigkeiten trug - neben der Vielfalt der Formulare und der Parteien, die Einträge in diese Dokumente vornahmen - gerade auch der Umstand bei, dass die Dokumente so lange gültig waren, wie es noch Platz für Einträge gab. Damit sollte ein Bild langfristigen Verhaltens gewährleistet werden, auch Aufwand, Kosten und Stempelgebühren dürften eine Rolle gespielt haben. Allerdings veränderten Personen im Laufe der Jahre ihr Aussehen, das in den Personenbeschreibungen ohnehin nur recht vage erfasst war. Erst 1920 mussten die Dienstkarten für Hausgehilfinnen, die nun anstelle der Dienstbotenbücher eingeführt wurden, Fotos aufweisen (vgl. StGBl. 1920/144), Ausweiskarten für gewerbliche Hilfsarbeiter*innen, die die Arbeitsbücher ab 1919 ersetzten, erst ab 1928 (vgl. BGBl. 1928/45). Besitzer*innen der Dokumente veränderten im Laufe ihres Lebens auch den 
Beruf oder beruflichen Status, die heimatrechtliche Zugehörigkeit, Frauen im Fall der Eheschließung ihren Namen. Solche Veränderungen wurden in den Dokumenten auch vermerkt, allerdings anscheinend oft in einer Weise, die den/die Betreffende* $n$ in den Verdacht der Fälschung bringen konnte (vgl. Abb. 2). Zum Schutz der Besitzer*innen sollte - so ein Erlass des Magistrates Wien - genau dokumentiert werden, welche Behörde die Änderungen vorgenommen hatte (Die Gemeinde-Verwaltung der Hauptund Residenzstadt Wien im Jahre 1904,1906, S. 404). Die Zuordnung eines Dokuments zu einer Person war und blieb aber problematisch.

Der Zwang, ein Dokument zu besitzen, wurde vielfach mit Sicherheit, Kontrolle und Vertrauen gerechtfertigt. Dokumente sollten der Feststellung der Identität dienen, sie boten aber auch Möglichkeiten, sich eine andere offizielle Identität bzw. die Identität eines/einer Anderen anzueignen, Kategorisierungen und Zuordnungen zu verändern (vgl. Higgs 2011, S. 10f.). Wie leicht dies gelang, wie sehr offizielle Dokumente die Wahrnehmung der Person tatsächlich beeinflussen und Vertrauen begründen konnten, ist schwer festzustellen. Die illegitime Nutzung von Dokumenten wird ja dort am ehesten greifbar, wo diese Wahrnehmungen korrigiert, die Erwartungen enttäuscht wurden.

In der zeitgenössischen Presse finden sich zahlreiche Berichte über Betrüger*innen, Dieb*innen und Mörder*innen, die ohne oder mit gestohlenen, falschen Dokumenten Zugang zu Haushalten fanden (Hedwig Ruß vor den Geschworenen, 1874). Dienstgeber*innen hätten die Dienstbotenbücher nicht ordentlich geprüft und deshalb Schaden erlitten. ${ }^{22}$ Gewerbeinspektoren bemängelten falsche Altersangaben in Ausweisdokumenten, die dazu dienten, das Verbot bzw. Beschränkungen der Kinder- und Jugendarbeit zu umgehen (vgl. Bericht der k.k. Gewerbe-Inspectoren z.B. 1896, S. XLVff; 1901, S. XCIf). In manchen Fällen war auch der in das Dokument eingetragene Beruf zweifelhaft oder strittig. ${ }^{23}$ Vagabunden konnten, so wurde zumindest geklagt, mit Arbeitsbüchern versehen leicht als arbeitswillige Wanderer erscheinen. Der sozialdemokratische Politiker Julius Deutsch schildert in seinen Lebenserinnerungen, wie er (und so mancher andere), um nach zu langer Arbeitslosigkeit der Gefahr zu entgehen, verhaftet und per Schub in die Heimatgemeinde befördert zu werden, eine falsche Arbeitsbestätigung in sein Arbeitsbuch eintragen ließ. Zwar ent-

22 Jodlbauer berichtet umgekehrt von einem Meister, der wohl die Dokumente, aber nicht den Inhaber selbst gesehen hat, Jodlbauer 1947-1948, S. 148.

23 Karl Ernst berichtet von der Korrektur der Berufsangabe in seinem Arbeitsbuch, Ernst 1912, S. 114ff. 
deckte der Gemeindevorsteher eines Grenzortes den Schwindel, dieser blieb jedoch nach Fürsprache von dessen Gattin ungeahndet (vgl. Deutsch 1960, S. 50f.). Die Leitmeritzer Zeitung berichtete 1901, dass die Gendarmerie in Waltirsche auf jemanden aufmerksam gemacht wurde, der in einem Gasthaus Tinte und Feder verlangte und sodann sein Arbeitsbuch fälschte. Gegenüber der Gendarmerie verwickelte sich der mehrfach vorbestrafte Mann dann in Widersprüche und gab an, „daß er dieses Arbeitsbuch fand, selbst keines besitze und zur, größeren Sicherheit' dasselbe fälschte." (Diebstahl im Auslande 1901, S. 7)

Die Sicherheit, die sich Benutzer*innen fremder oder gefälschter Dokumente erhofften, entspricht freilich nicht dem, was man gemeinhin als Sicherheit verstand oder verstehen mag, oder besser: was historisch als legitim und als Sicherheit durchgesetzt wurde. Aber Sicherheit, und wen oder was es zu schützen galt, was die richtige Identität und eine korrekte Angabe war - all das war Gegenstand des Konflikts, ein Produkt von Auseinandersetzungen und historisch veränderlich. Das wird etwa an einem außergewöhnlichen Fall besonders deutlich, der sich 1864 in verschiedenen Variationen in einer Reihe von Zeitungen findet, und den man aus heutiger Perspektive wohl unter völlig anderen Gesichtspunkten als die Zeitgenoss"innen interpretieren würde.

In der Steiermark war ein 25 Jahre alter Mann den Behörden vorgeführt worden, weil er sich im Besitz eines fremden Dienstbotenbuches befand. Der Bursche habe jahrelang bei verschiedenen Bauern zu deren Zufriedenheit als Knecht gedient. Ein Bauer hatte sogar geäußert, dass er nie einen so braven Burschen gehabt hätte, dass seine Pferde nie so fett und nett waren. Weit und breit wären die Mädchen in den schmucken und lustigen Burschen verliebt, dieser wäre aber stets „kalt und spröde gegen jede Dirn“ geblieben (Das Gespräch 1864). Einem anderen Bericht zufolge habe er die Arbeiten eines Knechtes, ja sogar das Fuhrwerk zur Zufriedenheit des Dienstgebers verrichtet, Tabak geraucht, Schnaps getrunken und sich auf Tanzböden mit Frauenzimmern unterhalten (Correspondenz 1864). Man fragte, heißt es in einer Version der Geschichte, den „hübschen Burschen, warum er sich noch nie als militärpflichtig gestellt habe." Er habe, meinte dieser, bislang keinen Auftrag erhalten. „Nun, so musst du dich jetzt stellen', hieß es, ,die Hosen herunter!' das wollte aber der Busche nicht, aber man fragte natürlich nicht lange und zog ihm die Hosen mit Gewalt herunter. Da sahen sich die Herren gegenseitig mit großen Augen an, denn der vermeintlich saubere Bauernknecht war - ein ,bildschönes Mädel' kugelrund und gewachsen wie eine Tanne." (Das Gespräch 1864) Diese aus heutiger Sicht offen gewalttätige Bloßstellung wurde in einer anderen Version relativiert: Der Bursche wäre, heißt es dort, da sein Körperbau etwas 
Weibliches verriet, ärztlich untersucht worden, wobei sich zeigte, dass „der Knecht wirklich weiblichen Geschlechtes sei.“ (Aus Mariazell 1864) ${ }^{24}$ Niemand, darin stimmten die verschiedenen Berichte überein, hatte etwas geahnt, auch nicht die „Mitknechte“, mit denen der Knecht/das Mädchen in einem Bette geschlafen hätte. Das Mädchen wollte, so heißt es, seiner eigenen Aussage zufolge lediglich als Knecht mehr Geld verdienen. Ein Autor zeigte sich erstaunt, dass Dirnen ihre Eitelkeit, Liebelei, Tändeleien aufgeben, auf alles verzichten würden, bloß um in Männerkleidern mehr zu verdienen (Das Gespräch 1864).25 Der Tenor der Berichte blieb das Erstaunen darüber, wie sich die Person, die „steierische Amazone“ (so die Presse, Mariazell. 2. Mai 1864, S. 5), deren Arbeitsleistung gelobt wurde, deren Verhalten und Aussehen offensichtlich über Monate hinweg tadellos und stimmig erschien, was aber letztlich nicht ins Gewicht fiel, auf unerlaubte Weise, mit Hilfe eines gestohlenen Dokuments eine - offiziell - falsche, korrekturbedürftige Identität aneignen konnte.

\section{Fazit: Unterschiede und Sicherheit(en) unterschiedlicher Art}

Ausweise wie Arbeits- und Dienstbotenbücher dokumentierten eine Reihe von Daten und Eigenschaften wie Name, Alter, Geschlecht, Aussehen, Beruf, heimatrechtliche Zugehörigkeit, Beschäftigungsverhältnisse etc. Sie wurden in verschiedenen Kontexten und Konstellationen zur Identifizierung, zur Kategorisierung und zur Etablierung von Unterschieden und (mehrdimensional bestimmten) Hierarchien benutzt: zwischen all jenen, die Dokumente dieser Art besitzen und mit sich führen mussten und jenen Staatsbürger*innen, die davon befreit waren oder solche Dokumente nicht erhalten konnten, aber auch zwischen gewerbliche/n Hilfsarbeiter"innen und Dienstbot"innen, Männern und Frauen, Minderjährige"n und Erwachsene"n, Fremde*n und Einheimische*n, Arbeitssuchende"n und vermeintlich arbeitsscheue"n Vagabund"innen. Sie sollten moralisch und politisch zuverlässige und vertrauenswürdige von gefährlichen Personen unterscheiden. Man hoffte, auf Grundlage des dokumentierten Vorle-

24 Diese Version findet sich auch in der Presse: Mariazell, 2. Mai. (Eine steierische Amazone.) 1864, S. 5.

25 Weniger Gewissheit denn Fragen finden sich in einem anderen Bericht: Das Mädchen hätte keine Auskunft gegeben, der Fall wäre an ein Untersuchungsgericht übergeben worden, dem es nun wohl gelinge dürfte, das Dunkel über die Herkunft desselben und den Grund der Verleugnung des Geschlechtes aufzuhellen. (Correspondenz 1864) 
bens einer Person, zukünftiges Verhalten einschätzen zu können. Nicht nur die in Frage stehenden Eigenschaften und Unterschiede waren dabei vielfältig. Verschiedene Sicherheiten, Schutz und Gefahren in der Perspektive der Behörden und Arbeit-/Dienstgeber"innen standen in den Debatten häufig denen von Dienstbot*innen und Arbeiter*innen gegenüber, ein Konsens wurde kaum erreicht. Fragt man nach 'der Sicherheit' allgemein oder im Zusammenhang mit Ausweisdokumenten, so läuft man leicht Gefahr, einen Anachronismus zu erzeugen. Nicht alle denkbaren Bedeutungen und Facetten von Sicherheit waren in den Debatten und Auseinandersetzungen des Untersuchungszeitraums und in jedem Kontext gleichermaßen präsent, nicht in jeder Hinsicht wurde überhaupt explizit von 'Sicherheit' gesprochen. Während, innere Sicherheit', ,Sicherheit des Eigentums und der Person' bereits im frühen 19. Jahrhundert stehende Wendungen waren, ${ }^{26}$ etablierte sich erst um die Wende zum 20. Jahrhundert auch „soziale Sicherheit“ als Terminus und als Vorstellung, auf die in diesen Debatten Bezug genommen wurde. Dabei lassen sich an Arbeitsund Dienstbotenbüchern neben Unterschieden der Arbeitsverhältnisse samt der damit verknüpften sozialen und politischen Rechte und Pflichten auch geschlechts- und altersspezifische Aspekte festmachen. Schutz und Rechte der Arbeiter*innen wurden eher zum politischen Thema als jene der meist weiblichen Dienstbot*innen oder des Gesindes. Die Perspektiven und Positionen der Arbeiter*innen - zumindest der politisch organisierten - lassen sich eher noch als die der wesentlich schlechter organisierten Dienstbot*innen rekonstruieren.

Arbeits- und Dienstbotenbücher waren wohl vielen Arbeiter*innen und Dienstbot"innen verhasst, sie wurden häufig als Symbol der Erniedrigung, der Klassenunterschiede und als Instrument der Unterdrückung und der Willkür der Arbeit- und Dienstgeber*innen betrachtet. Die entsprechenden Bestimmungen wurden boykottiert, offen bekämpft, aber auch oft hingenommen. Die Dokumente wurden in vielfältiger Weise benutzt und zum eigenen Vorteil gewendet oder missbraucht, von denen, die sie besitzen und vorweisen sollten wie auch von Behörden und Arbeit-/Dienstgeber*innen. Die konsequente Durchsetzung der Arbeits- und Identitätsdokumente in dieser Form scheiterte wohl letztendlich daran, dass kein Konsens über Form, Nutzen und legitime Gebrauchsweisen der Doku-

26 Ich beziehe mich hier etwa auf die digitalisierten Zeitungsbestände der österreichischen Nationalbibliothek (http://anno.onb.ac.at/), die es erlauben, das Aufkommen solcher Begriffe zumindest in den dort erfassten Publikationen zu untersuchen. 
mente, über erstrebenswerte Sicherheiten und zu vermeidende Gefahren hergestellt wurde bzw. hergestellt werden konnte. ${ }^{27}$

\section{Literatur}

About, Ilsen/Brown, James/Lonergan, Gayle (Hrsg.) (2013): Identification and Registration Practices in Transnational Perspective. People, Papers and Practices. Houndmills, New York: Palgrave Macmillan.

Althaus, Andrea (2010): Lebensverhältnisse von Dienstmädchen und Hausgehilfinnen im 19. und 20. Jahrhundert. In: Dies. (Hg.): Mit Kochlöffel und Staubwedel. Erzählungen aus dem Dienstmädchenalltag (= Damit es nicht verlorengeht ... 62), Wien, Köln, Weimar: Böhlau, S. 275-292.

Antrag des Abgeordneten Smitka, Palme und Genossen (1911), betreffend die Beseitigung der Arbeitsbücher (Entlaßscheine, Seedienstbücher). Stenographische Protokolle über die Sitzungen des Hauses der Abgeordneten des österreichischen Reichsrathes, XXI Session, Beilage 70. Wien: Kaiserlich-königliche Hofund Staatsdruckerei.

Arbeitsbücher (1912). In: Arbeiter-Zeitung XXIV, Nr. 143, 26.5.1912, S. 13.

Aus Mariazell (1864). In: Fremden Blatt XVIII, Nr. 124, 5.5.1864, S. 4.

Bericht der k.k. Gewerbe-Inspectoren über ihre Amtsthätigkeit (1884-1916). Wien: Druck und Verlag der kaiserlich-königlichen Staatsdruckerei.

Bericht des sozialpolitischen Ausschusses (1913) betreffend eine über Antrag des Abgeordneten Dr. Freißler im Sinne des $\$ 33$ G.O. an das Haus zu stellende Vorfrage, „ob die Regierung aufzufordern sei, eine Vorlage einzubringen, welche den Erlaß des Arbeitsbuches für erwachsene Arbeiter durch eine geeignete Legitimation vorsieht. 1860 der Beilagen zu den stenogr. Protokollen des Abgeordnetenhauses XXI Session.

Berufsstatistik nach den Ergebnissen der Volkszählung vom 31. Dezember 1910 (1916). In: Österreichische Statistik NF Bd. 3, H. 1.

Breckenridge, Keith/Szreter, Simon (Hrsg.) (2012): Registration and Recognition. Documenting the Person in World History. Oxford: Blackwell Publishing Ltd.

Burger, Hannelore (2014): Heimatrecht und Staatsbürgerschaft österreichischer Juden. Vom Ende des 18. Jahrhunderts bis in die Gegenwart. Wien, Köln, Graz: Böhlau.

Caplan, Jane/Torpey, John (Hsg.) (2001): Documenting Individual Identity. The Development of State Practices in the Modern World. Princeton, Oxford: Princeton University Press.

27 In diesem Text wurden verschiedene Positionen und Konstellationen nur sehr allgemein skizziert. Die verschiedenen Gebrauchsweisen der Dokumente, Interaktionen und Auseinandersetzungen systematisch und in verschiedenen Kontexten zu rekonstruieren und zu untersuchen, ist Ziel meiner weiteren Forschung. 
Conze, Eckart (2018): Geschichte der Sicherheit: Entwicklung - Themen - Perspektiven. Göttingen: Vandenhoeck \& Ruprecht.

Conze, Werner (1984): Sicherheit, Schutz. In: Geschichtliche Grundbegriffe. Historisches Lexikon zur politisch-sozialen Sprache in Deutschland. Bd. 5, hrsg. von Brunner, Otto/Conze, Werner/Koselleck, Reinhart. Stuttgart: Klett-Cotta Verlag, S. 831-862.

Correspondenz aus der Provinz. Maria-Zell am 2. Mai. (Auch ein ,Peter Thomas.') (1864). In: Grazer Zeitung Nr. 104, 8.5.1864, S. 3.

Daase, Christopher (2012): Die Historisierung der Sicherheit. Anmerkungen zur historischen Sicherheitsforschung aus politikwissenschaftlicher Sicht. In: Geschichte und Gesellschaft 38, H. 3, S. 387-405.

Das Gespräch am Wirthshaustische. Der Wirth von Hetzendorf „Michl Glattweg“ und seine Gäste (1864). In: Gemeinde-Zeitung: unabhängiges politisches Journal III, Nr. 19, 12.5.1864, S. 10.

Deak, John (2015): Forging a Multinational State: State Making in Imperial Austria From the Enlightenment to the First World War. Stanford: Stanford University Press.

Deutsch, Julius (1960): Ein weiter Weg. Lebenserinnerungen. Zürich, Leipzig, Wien: Amaltea.

Die Abschaffung des Arbeitsbuches auf Grund des $₫ 14$. (1914). In: Allgemeiner Tiroler Anzeiger VII, Nr. 162, 18.7.1914, S. 17.

Die Aufhebung der Arbeitsbücher (1919). In: Deutsches Volksblatt 31, Nr. 10800, 29.1.1919, S. 7.

Die Dienstbotenfrage in Wien (1892). In: Die Presse 45, Nr. 33, 2.2.1892, S. 9-11.

Die Gemeinde-Verwaltung der Haupt- und Residenzstadt Wien im Jahre 1904 (1906). Wien: Gerlach \& Wiedling.

Diebstahl im Auslande (1901). In: Leitmeritzer Zeitung 31, Nr. 74, 21.9.1901, S. 7.

Ebenhoch, Alfred/Pernerstorfer, Engelbert (1893) (Hrsg.): Stenographisches Protokoll der Gewerbe-Enquête im österreichischen Abgeordnetenhause sammt geschichtlicher Einleitung und Anhang. Wien: Kaiserlich-königliche Hof- und Staatsdruckerei.

Ebenhoch, Alfred (1893): Die mündliche Gewerbe-Enquête im österreichischen Parlamente und die Gewerbe-Reform in Oesterreich. O.O. [Wels: PreßvereinsDruckerei].

Ehrenfreund, Edm. O./Mráz, Franz (1908): Handbuch für politische und Gerichtsbehörden, Advokaten, sowie für das Haus. Wien: Manz.

Erlass der k.k. Statthalterei für Oberösterreich vom 9.1.1865 Z 4909/Präs., betreffend die Beschränkung in Ausstellung der Legitimations-Karten für Dienstboten und gewerbliche Hilfsarbeiter. Gesetz- und Verordnungsblatt für das Erzherzogthum Österreich 1865/1.

Ernst, Karl (1912): Aus dem Leben eines Handwerksburschen. Mit einem Geleitwort von Heinrich Hansjakob. Neustadt im Schwarzwald: Karl Wehrle (Erstauflage 1911). 
Ertl, Moriz (1886): Bericht der k.k. Gewerbeinspektoren über ihre Amtsthätigkeit im Jahre 1884. Wien 1885. In: Statistische Monatschrift XII, S. 54- 59.

Fahrmeir, Andreas (2019): Conclusion. Cities and States: Papers and Walls. In: Greefs, Hilde/ Winter, Anne (Hg.): Migration Policies and Materialities of Identification in European Cities. Papers and Gates, 1500s to 1930s. New York, London: Routledge, S. 289-306.

Greefs, Hilde/Winter, Anne (Hrsg.) (2019): Migration Policies and Materialities of Identification in European Cities. Papers and Gates, 1500s to 1930s. New York, London: Routledge.

Groebner, Valentin (2004): Der Schein der Person. Steckbrief, Ausweis und Kontrolle im Europa des Mittelalters. München: Beck.

Hart, Keith (1973): Informal Income Opportunities and Urban Employment in Ghana. In: The Journal of Modern African Studies II, I, S. 61-89.

Heilinger, Alois (1909): Österreichisches Gewerberecht. Kommentar der Gewerbeordnung. Dritte, vom Verfasser durchgesehene, die neueste Gesetzgebung und Judikatur berücksichtigende Auflage, bearbeitet von Michael Plachy und Alois Tischler. Wien: Manz.

Heindl, Waltraud/Saurer, Edith (2000) (Hrsg.): Grenze und Staat. Paßwesen, Staatsbürgerschaft, Heimatrecht und Fremdengesetzgebung in der österreichischen Monarchie 1750-1867. Wien: Böhlau.

Heller, Emil (1908): Das österreichische Gewerberecht mit Berücksichtigung der Gewerbenovelle vom 5. Februar 1907, RGBl. Nr. 26. Systematische Darstellung für die Praxis. Wien: Manz.

Higgs, Edward (2011): Identifying the English. A History of Personal Identification 1500 to the Present. London, New York: Continuum.

Hoerder, Dirk/van Nederveen Meerkerk, Elise/Neunsinger, Silke (Hrsg.) (2015): Towards a Global History of Domestic and Caregiving Workers, Leiden, Boston: Brill.

Ingwer, Isidor (1895): Der sogenannte Arbeitsvertrag. Eine sozialpolitische Studie. Wien: Verl. d. "Österreichischen Metallarbeiter".

Ingwer, Isidor (1912): Zwei Fesseln des Koalitionsrechtes. Wien: Österr. Metallarbeiterverband.

Jodlbauer, Josef (1947-1948): Ein Mensch zieht in die Welt/Ein Mensch sieht die Welt. Selbstbiographie eines Altösterreichers. Typoskript. Dokumentation lebensgeschichtlicher Aufzeichnungen, Universität Wien.

Keiser, Thorsten (2013): Vertragszwang und Vertragsfreiheit im Recht der Arbeit von der frühen Neuzeit bis in die Moderne. Frankfurt am Main: Vittorio Klostermann.

Krautsieder, Karl (1926): Die Verläßlichkeit der Ausweisdokumente. In: Öffentliche Sicherheit 6, Nr. 13, S. 4.

Kundmachung 231 (1828). In: Seiner k.k. Majestät Franz des Ersten politische Gesetze und Verordnungen für sämmtliche Provinzen des Österreichischen Kaiserstaates, mit Ausnahme von Ungarn und Siebenbürgen Wien: k.k. Hof- u. StaatsAeralial-Druckerei, S. 283-289. 
Landriani, Martino Sacchi (2019): Rethinking the livret d'ouvriers: Time, Space and 'Free' Labor in Nineteenth Century France. In: Labour History 60, Nr. 6, S. 854-864.

Lehnert, Katrin (2017): Die Un-Ordnung der Grenze. Mobiler Alltag zwischen Sachsen und Böhmen und die Produktion von Migration im 19. Jahrhundert. Leipzig: Leipziger Universitätsverlag GmbH.

Linne, Karsten (2014): Von der Arbeitsvermittlung zum „Arbeitseinsatz“. Zum Wandel der Arbeitsverwaltung 1933-1945. In: Buggeln, Marc/Wildt, Michael (Hrsg.): Arbeit im Nationalsozialismus. Berlin, Boston, Oldenbourg, S. 53-70.

Lyon, David (2009): Identifying Citizens. ID Cards as Surveillance. Cambridge.

Mariazell, 2. Mai. (Eine steierische Amazone.) (1864). In: Die Presse 17, Nr.124, 5.5.1864, S. 5 .

Mayerhofer, Ernst (1897): Ernst Mayerhofer's Handbuch für den politischen Verwaltungsdienst in den im Reichsrathe vertretenen Königreichen und Ländern mit besonderer Berücksichtigung der diesen Ländern gemeinsamen Gesetze und Verordnungen. Bd. 3, Wien: Manz, S. 536-582.

Morgenstern, Hugo (1902): Gesindewesen und Gesinderecht in Österreich. (=Mittheilungen des k.k. arbeitsstatistischen Amtes im Handelsministerium. 3. Heft.) Wien: Hölder.

Morgenstern, Hugo (1912): Österreichisches Gesinderecht. Handbuch und systematische Darstellung des gesamten, in Österreich geltenden Gesinderechtes nach den bestehenden 24 Dienstbotenordnungen einschließlich der Gesindepolitik und des Verwaltungsverfahrens in Gesindestreitigkeiten. Wien: Hölder.

Patent vom 24. Februar 1827. Aufhebung der Kundschaften, Zeugnisse, Wanderpässe für Handwerksgesellen und Arbeiter; Einführung der Wanderbücher (1829). In: Seiner k.k. Majestät Franz des Ersten politische Gesetze und Verordnungen für sämmtliche Provinzen des Österreichischen Kaiserstaates, mit Ausnahme von Ungarn und Siebenbürgen. Wien: k.k. Hof- u. Staats-Aeralial-Druckerei, S. 231f.

Pierson, Thomas (2016): Das Gesinde und die Herausbildung moderner Privatrechtsprinzipien. Frankfurt am Main: Vittorio Klostermann.

Polizeidirektion Wien (1920): Das polizeiliche Meldewesen. Wien: Selbstverlag d. Polizeidirektion.

Popp, Adelheit (1912): Haussklavinnen. Ein Beitrag zur Lage der Dienstmädchen. Wien: Brand.

Reinecke, Christiane (2010): Grenzen der Freizügigkeit. Migrationskontrolle in Großbritannien und Deutschland, 1880-1930. Berlin, Boston: Oldenbourg.

Richter, Jessica (2017): Die Produktion besonderer Arbeitskräfte. Auseinandersetzungen um den häuslichen Dienst in Österreich (Ende des 19. Jahrhunderts bis 1938). Unpublizierte Dissertation. Universität Wien.

Rosenbaum, Ad. (1908): Über einige paßpolizeiliche Fragen. In: Österreichische Zeitschrift für Verwaltung XLI, Nr. 43, S. 175f; XLI, Nr. 44, S. 179f; XLI, Nr. 45, S. $183 \mathrm{ff}$. 
Ruß, Hedwig (1874): Vor den Geschworenen. In: Neues Fremden-Blatt 10, Nr. 141, 23.5.1874, S. 1-3.

Sammlungen der Entscheidungen der k.k. Gewerbegerichte (1900-1920). Wien: Kaiserlich-königliche Hof- und Staatsdruckerei.

Sarti, Raffaella (2014): Historians, Social Scientists, Servants, and Domestic Workers: Fifty Years of Research on Domestic Care Work. In: International Review of Social History 59, H. 2, S. 279-314.

Sitzungs-Protokolle des ständigen Arbeitsbeitrates (1914). 1913 und 1914. (33. bis 35. Sitzung). Wien: k.k. Hof- und Staatsdruckerei.

Stanziani, Alessandro (2009): The Legal Status of Labour from the Seventeenth to the Nineteenth Century: Russia in a Comparative European Perspective. In: International Review of Social History 54, H. 3, S. 359-389.

Stenographische Protokolle über die Sitzungen des Hauses der Abgeordneten des österreichischen Reichsrathes, XXI Session, 161. Sitzung, 20.6.1913. Wien: k.k. Hof- und Staatsdruckerei.

Stieda, Wilhelm (1890): Arbeitsbuch. In: Conrad, Johannes/Elster, Ludwig/Lexis, Wilhelm/Loening, Edgar (Hrsg.): Handwörterbuch der Staatswissenschaften.. Bd 1. Jena: Fischer, S. 598-604.

Stopp, Klaus (1982): Die Handwerkskundschaften mit Ortsansichten. Stuttgart: Hiersemann.

Tálos, Emmerich/Wörister, Karl (1994): Soziale Sicherung im Sozialstaat Österreich. Entwicklung - Herausforderungen - Strukturen. Baden-Baden: Nomos.

Torpey, John (2000): The Invention of the Passport. Surveillance, Citizenship and the State. Cambridge: Cambridge University Press.

Treu, fleißig, sittlich (1879). In: Neue Freie Presse Nr. 5161, 9.1.1879, S. 6.

Unsere Dienstboten-Misère (1876). In: Leitmeritzer Wochenblatt 21, Nr. 23, 22.3. 1876, S. 1.

Verkauf, Leo (1905): Arbeitsvertrag. In: Österreichisches Staatswörterbuch. Handbuch des gesamten österreichischen öffentlichen Rechtes. Herausgegeben unter Mitwirkung zahlreicher Fachmänner von Mischler, Ernst/Ulbrich, Josef. 2., wesentlich umgearbeitete Auflage. Bd. 1, Wien: Hölder, S. 149-187.

Vormbaum, Thomas (2014): Gesinderecht und Politik im 19. Jahrhundert. In: Scheiwe, Kirsten/Krawietz, Johanna (Hsg.): (K)Eine Arbeit wie jede andere? Die Regulierung von Arbeit im Privathaushalt, Berlin, Boston: De Gruyter, S. 23-40.

Vormbaum, Thomas (1980): Politik und Gesinderecht im 19. Jahrhundert (vornehmlich in Preußen 1810-1918). Berlin, Duncker \& Humblot.

Wadauer, Sigrid (2015): Tramping in Search of Work. Practices of Wayfarers and of Authorities (Austria 1880-1938). In: Wadauer, Sigrid/Buchner, Thomas/ Mejstrik, Alexander (Hrsg.): The History of Labour Intermediation. Institutions and Finding Employment in the Nineteenth and Early Twentieth Centuries. New York, Oxford: Berghahn, S. 286-334.

Weiss, Karolina (1907) Lebenserinnerungen, Typoskript, Dokumentation lebensgeschichtlicher Aufzeichnungen, Universität Wien. 
Werner, Stefanie/Degner, Harald/Adamo, Mark (2011): Hitlers gläserne Arbeitskräfte. Das Arbeitsbuch als Quelle von Mikrodaten für die historische Arbeitsmarktforschung. In: Jahrbuch für Wirtschaftsgeschichte 52, 2, S. 175-192.

Winter, Fritz (1900): Statistisches. In: Dokumente der Frauen 2, S. 584-589.

Vermischtes (1893). In: Arbeiterinnen-Zeitung 2, Nr. 6, 17.3.1893, S. 6-8.

Abkürzungen

BGBl. Bundesgesetzblatt

LGBl. Landesgesetzblatt

RGBl. Reichsgesetzblatt

StGBl. Staatsgesetzblatt

WStLA Wiener Stadt- und Landesarchiv 
